\title{
Justicia espacial y bienes comunes en el Territorial Rural de Medellín
}

\author{
SPATIAL JUSTICE AND COMMONS IN THE MEDELLIN'S RURAL TERRITORY
}

Torrejón, E. *1 Egio Rubio, C. *2

eryka.torrejon@udea.edu.co

Recibido: 01/11/2019 Aceptado: 15/12/2019

\section{Resumen}

Este trabajo presenta los resultados de una investigación donde se usa el término de justicia espacial, sentido del lugar y bienes comunes como ejes teóricos. Se desarrolla con metodologías cualitativas en el contexto de las zonas rurales de Medellín. Su objetivo es identificar y clasificar los actores sociales y sus discursos en la política de ordenamiento territorial durante el período 2009-2019. Los resultados que se encuentran son (i) una gran diversidad de actores sociales, (ii) un alto nivel de dispersión de acciones en todo el territorio rural y (iii) unos sentidos de lugar asociados a identidades locales en transición entre una ruralidad tradicional y otra moderna.

Palabras claves: justicia espacial, espacios rurales, bienes comunes, Medellín

\begin{abstract}
This paper presents the results of an investigation where space justice, the senses places and commons are used as theoretical concepts. It develops with qualitative methodologies in the context of rural areas of Medellin. Their goal is to identify and classify the social actors and their discourses involved in the policies for the period 2009-2014. The results found are (i) a wide range of social actors, (ii) a high level of dispersion of actions in all rural territories a (iii) a sense of place associated with changing local identities of a traditional rurality and modern.
\end{abstract}

Key word: space justice, local actor, rural space, Medellin city.

\section{Presentación}

El artículo se compone en su primera parte de una breve descripción de los antecedentes y del proceso de ordenamiento territorial rural $^{3}$ de la ciudad. En su segunda parte presenta el contexto y la metodología bajo el enfoque de la investigación cualitativa. En la tercera parte, los resultados de la investigación referidos a la clasificación de actores y discursos en el escenario de la formulación, debate, aprobación y seguimiento del Plan de Ordenamiento Territorial POT (Acuerdo 48 del 2014) y, finalmente las reflexiones.

\footnotetext{
1 *_ Universidad de Antioquia (Colombia) - Universidad Autónoma de Barcelona (España)

2 **_Universidad de Murcia (España)

${ }^{3}$ En el contexto local se les denomina corregimientos y corresponde a la forma administrativa para reconocer las cinco zonas rurales de la ciudad de Medellín: Altavista, Santa Elena, San Antonio de Prado, San Cristóbal y San Sebastián de Palmitas.
}

Tekoporá ${ }^{\circledR}$. Centro Universitario de la Región Este. Universidad de la República (C) Torrejón (2020)

Este es un artículo de Acceso Abierto distribuido bajo licencia Creative Commons (CC BY NC 4.0) 
Los resultados indican que, (i) hay una gran diversidad de actores sociales y discursos (ii) un alto nivel de dispersión de acciones y (iii) unas identidades locales en constante cambio y reconstrucción asociadas, casi siempre, a nuevas formas de ruralidad. Las reflexiones, como parte final de este artículo, vinculan la justicia espacial como práctica política de los colectivos sociales de la ciudad de Medellín y, como una de las formas para defender los recursos naturales como bienes públicos (inalienables, intransferibles e inembargables) desde las experiencias locales centradas en la defensa de un territorio rural campesino, a implementar como Distrito Rural Campesino4 DRC, en el Plan de Ordenamiento Territorial (Acuerdo 048 del 2014, artículo 485)

\section{Antecedentes}

En la literatura especializada de las zonas rurales de Medellín se presentan las visiones nostálgicas de la vida campesina y otras visiones vinculadas a los procesos de metropolización regional, según los aportes de Arango (2008), Cadavid (2009), García y Gálvez (2011), Agudelo (2012) y Castaño y Agudelo (2014).

También estos aportes, indican la necesidad de regular los usos del suelo de las zonas rurales frente a la expansión urbana de la ciudad, se subraya en particular el uso de elementos emblemáticos de una cultura campesina andina como valores locales y, en ocasiones en contracorriente $\mathrm{o}$, en procesos de adaptación con el crecimiento económico y de infraestructura que ha tenido la ciudad en las últimas dos décadas.

La producción académica alrededor de las zonas rurales de Medellín es una evidencia de que el tema de la política de ordenamiento territorial de las zonas rurales ha escalado posición, primero frente a los actores sociales implicados y, luego, como tema de relevancia para los colectivos académicos interesados en los estudios urbano-regionales. En forma retrospectiva, y al margen de un estado del arte (como tarea pendiente para otro momento de la investigación social), sobresale el proceso mediante el cual la ciudad de Medellín formuló y decretó una política de ordenamiento territorial rural.

Esta política se enmarca en el Plan de Ordenamiento Territorial POT (Acuerdo Municipal 062 de 1999), sin embargo, como se presenta en las siguientes etapas, ésta nace a partir de las ausencias técnicas y de enfoque para abordar un territorio rural tan extenso

\footnotetext{
4 Zona especial de reserva agraria para la seguridad alimentaria de la población con manejo apropiado del ambiente y uso social del territorio (Municipio de Medellín, 2005).
} 
como complejo y avanza en el tiempo en la construcción de una contundente y multivariada plataforma de instrumentos de planificación con incidencia en las zonas rurales de Medellín (Cadavid, 2009; Agudelo, 2012). Así, en este trabajo, los datos -documentos, sociograma y entrevistas- han permitido delimitar tres grandes momentos en la política de ordenamiento territorial con énfasis en lo rural:

La primera se enmarca en los episodios donde la administración municipal, en desarrollo de la Ley 388 de 1997, formula el primer plan de ordenamiento territorial. Ese momento y el documento que regiría la ordenación del territorio municipal se focalizó en el área urbana. Casi el 70\% del territorio municipal quedó o estuvo potencialmente aislado de las medidas e instrumentos de La Ley 388. Este momento, sin duda, permitió que, para los años 2004 a 2006, la revisión del POT se plantease una medida contundente para vincular este $70 \%$ del territorio rural en forma integral y compensatoria proporcionalmente a su tamaño y funciones espaciales decretadas -servicios ambientales y zonas de expansión urbana-.

La segunda etapa que se identifica parte de 2006, cuando tiene lugar la presentación del Plan de Estrategias Corregimentales PLAN ECO (Municipio de Medellín, 2005). Este plan logra vincular y posicionar las zonas rurales de Medellín en el ejercicio del ordenamiento territorial y se generan otros instrumentos a lo largo de los siguientes años: Planes Especiales de Ordenamiento Corregimental, Planes de Desarrollo Rural Sostenible, Planes Corregimentales de Cultura, Planes de Desarrollo Local de los Corregimientos y la Estrategia de Hábitat Rural Sostenible, entre otros.

La tercera etapa (2008-2019) se caracteriza ahora, no por la ausencia de medidas, sino por la alta proliferación de estas en la regulación del territorio rural y, en forma central, por la formulación, debate, aprobación y seguimiento del Plan de Ordenamiento Territorial (Acuerdo Municipal 48 el 2014). El nuevo POT incluye una serie variada de artículos con efectos en el ordenamiento territorial rural y genera múltiples polémicas en los diferentes colectivos de la ciudad debido a las implicaciones de este articulado para el futuro de las poblaciones tradicionales y las no tan tradicionales de las zonas rurales. Esta tercera etapa será contundente y decisiva para el ordenamiento territorial de la ciudad bajo los consensos o disensos en la implementación del POT y la figura del Distrito Rural Campesino (artículo 485)

En resumen, se encuentra que, la política de ordenamiento territorial de las zonas rurales de Medellín es reciente en cuanto a su formulación y aplicación (menos de una 
década) y, con el conjunto de etapas que se presentan, se puede identificar un territorio rural donde los instrumentos de ordenación territorial proliferan y donde su coordinación y enfoque en muchas ocasiones es centro de debate entre las instituciones, los colectivos sociales y la población rural.

La política de ordenamiento territorial también tiene el reto de enfrentar los cambios 5 de las zonas rurales frente a los procesos de globalización de la ciudad contemporánea, estos retos exigen una planeación de largo plazo con consensos básicos a nivel técnico, ético, social, económico y ambiental.

En el ordenamiento territorial rural de Medellín y los consensos básicos del modelo de desarrollo se presenta dos elementos constitutivos para las ciudades contemporáneas, el primero se refiere a la justicia espacial como un correlato del derecho a la ciudad y, el debate por los bienes comunes como forma de reconstruir la interdependencia entre el campo y ciudad.

Promovida por el derecho a la ciudad (Lefebvre, 1978), la justicia espacial (Soja, 2010), rescata las prácticas colectivas en el espacio, la capacidad de transformar el espacio social y en especial, la posibilidad creativa de imaginar otras formas de vida local. La justicia espacial valora en forma central, además de esta capacidad transformadora, las acciones políticas en el espacio, es decir, rescata las habilidades de los colectivos para planear, organizar y construir sus propias iniciativas locales.

Desde la visión trialéctica del espacio de Lefebvre (1983) el espacio vivido es asumido como vida cotidiana y es en la vida cotidiana donde el espacio se transforma en lugar, en tanto que el sentido global del lugar se determina por la relación entre escalas, y éstas, las escalas, pueden cambiar acorde a los actores sociales. Las identidades locales están vinculadas a la producción de un espacio a escala humana (espacio vivido), revirtiendo o reconstruyendo lo que Massey denomina, la geometría del poder (Massey, citado en Albet y Bénach, 2012, p.236).

Desde otra óptica, Capel (2003) indica que, los recursos naturales como bienes comunes se vinculan con la apropiación social del espacio y, por tanto, hay una tensión permanente entre apropiación privada y apropiación colectiva. Varias experiencias de uso colectivo de los recursos naturales han demostrado que la gestión colectiva del agua, los

5 Para describir estos cambios aparecen términos como urbanismo rural, nueva ruralidad, rururbanización, peri urbanización, ciudad difusa, entre otros neologismos. 
bosques o subproductos del bosque y la fauna han sido eficaces y sostenibles en el tiempo, precisando así que, la relación de bienes comunes (en el sentido de recursos naturales como bienes públicos para este texto) es compatible y regular en la cogestión del territorio por parte de comunidades raizales o, también en experiencias dentro de sociedades industrializadas o post industrializadas.

Autores como Boof (1996), Escobar (1999a, 1999b), Acosta (2010, 2104), Gudynas (2011) van a enfatizar en las diferentes experiencias de cogestión de los recursos naturales por parte de colectivos sociales como los indígenas y afrodescendientes (en Colombia, Perú, Ecuador, Bolivia y México), subrayando, además, las visiones de integración con la naturaleza en contraste con las visiones occidentales de exteriorización de la naturaleza como objeto, producto o mercancía. La naturaleza para estos pueblos ancestrales hace parte integral de sus cosmogonías y por tanto su vida cotidiana y espiritual se determina en y desde ésta (la naturaleza).

Así, los debates sobre los bienes comunes tienen correlatos desde la ecología política que los anteceden y abundan los casos de cogestión de los recursos naturales en Sur y Meso América, demostrando las posibilidades de las comunidades locales para su eco desarrollo sin detrimento de la privatización o extracción de los recursos naturales, sean estas comunidades ancestrales $\mathrm{y} / \mathrm{o}$ experiencias organizativas en sociedades industrializadas o post industrializadas.

Los autores, desde diferentes enfoques coinciden en estimar que, la producción social del espacio es una práctica cotidiana y colectiva (Lefebvre, 1983; Soja, 2010) donde se materializa el derecho a la ciudad y la justicia espacial como capacidad de transformación. Y, la cogestión de los bienes comunes (entendidos como recursos naturales) es a la vez, una opción y ejercicio de poder local frente a la tendencia de extracción y privatización de recursos naturales y culturales.

Con la justicia espacial y los bienes comunes se integran dos elementos sustanciales para el análisis del ordenamiento territorial rural de Medellín, el primero se refiera a los derechos colectivos sobre los territorios y, a la conciencia espacial vinculada ahora, por un sentido particular y universal, es decir, un sentido global del lugar donde se reconstruyen las identidades locales justo por su posición y ubicación local y global. 


\section{Contexto}

Medellín se ubica en la parte central del Valle de Aburrá en el Departamento de Antioquia, zona noreste de Colombia (Figura 1) y a su vez, el Valle de Aburrá se encuentra en el centro de los Andes Latinoamericanos, entre las cordilleras Central y Este, dos de las tres ramificaciones en que se dividen los Andes al llegar al territorio colombiano.

Medellín, Bello, Copacabana, Girardota y Barbosa al norte y Envigado, Itagüí, La Estrella y Sabaneta hacia el sur, conforman lo que se ha denominado el Área Metropolitana del Valle de Aburrá (Municipio de Medellín, 2006).

El Municipio de Medellín cuenta con una zona urbana $(110 \mathrm{~km} 2)$ y una zona rural conformada por cinco corregimientos $(270 \mathrm{~km} 2)$. La zona urbana contiene dieciséis comunas, equivalente a 29,5\% de la extensión total de Medellín. La zona rural cubre el 70,5\% de su territorio y se conforma por los corregimientos de Santa Elena, San Antonio de Prado, Altavista, San Sebastián de Palmitas y San Cristóbal (Municipio de Medellín, 2006).

Altavista: Se ubica al oeste de la ciudad, tiene una extensión de $27.4 \mathrm{Km} 2$ colindando con las comunas de La América y Belén lo que influye en su articulación y dependencia con dinámicas urbanas como la construcción de vivienda e infraestructura pues, en su territorio se asientan empresas para la extracción de arena, arcilla y triturado de rocas (Municipio de Medellín, 2005).

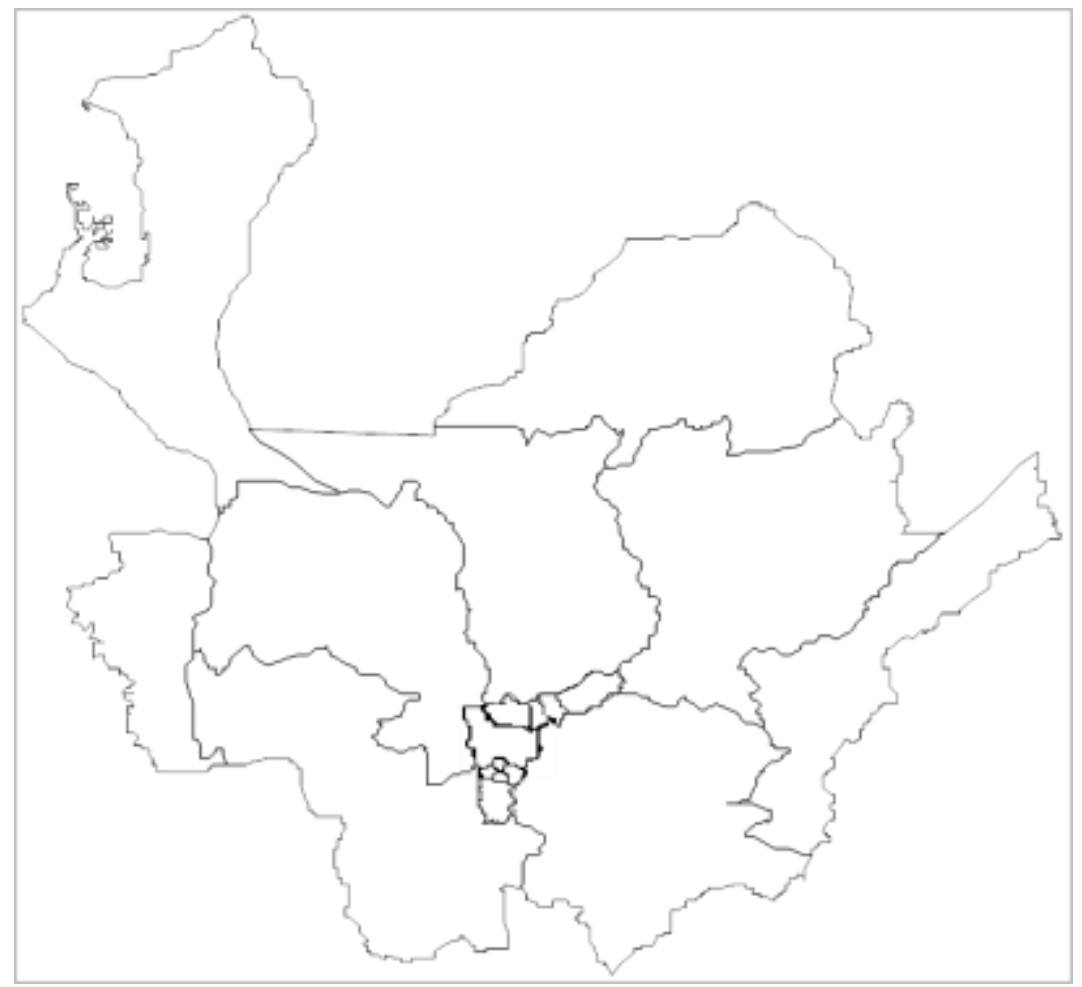

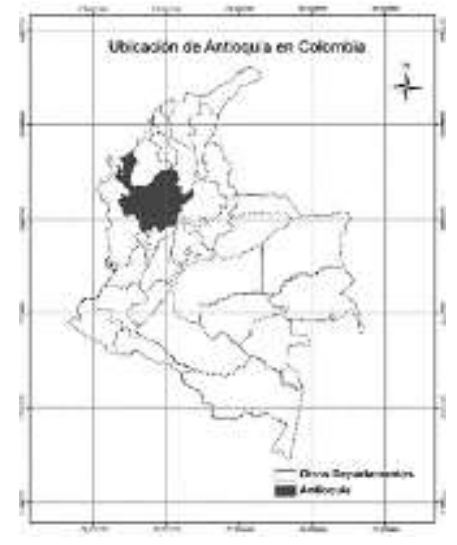

Figura 1. Ubicación de Medellín en Antioquia y Antioquia en Colombia en la parte superior de la figura. Elaboración propia. Fuente: Municipio de Medellín, 2005. 
San Antonio de Prado: Se ubica al sur oeste de la ciudad, tiene una extensión $60.4 \mathrm{Km}^{2}$ Su economía se centra en la explotación pecuaria y las plantaciones forestales. A partir de 1980 se incrementó el uso para vivienda social, duplicando la tasa de crecimiento y el área urbana de su cabecera corregimental, gran parte de su suelo es clasificado como suelo de expansión (Municipio de Medellín, 2005).

Santa Elena: Tiene una extensión de $70.4 \mathrm{Km}^{2}$, se ubica al noreste de la ciudad, se ha vinculado desde la primera mitad del siglo XX al crecimiento de Medellín para la producción de agua potable a partir de la construcción del Embalse de Piedra Blancas lo que impulso a la vez, zonas boscosas que aún se conservan. Actualmente, gran parte de su territorio hace parte del Parque Regional Arví, figura estimada para la conservación de espacio verde público del área Metropolitana del Valle de Aburrá (Municipio de Medellín, 2005).

San Cristóbal: Tiene una extensión de $49.5 \mathrm{Km}^{2}$, se ubica al oeste de la ciudad de Medellín y es de gran importancia en la producción de hortalizas y verduras en contraste con un fuerte crecimiento de urbanización precaria en sus bordes desde los años 1990. Las obras de infraestructura vial para reducir el tiempo de conexión del Valle de Aburrá hacia la salida al mar (Golfo de Urabá) en conjunto con la expansión urbana de la zona norte de la ciudad (la zona geográfica más densamente poblada) han generado cambios en el uso del suelo y en el perfil poblacional de este corregimiento.

San Sebastián de Palmitas: Se localiza hacia la zona noroeste de Medellín, con una extensión territorial de $57.5 \mathrm{Km}^{2}$ y a una distancia de $32 \mathrm{Km}$ de Medellín. Este corregimiento, también se ha considerado como un corredor para la comunicación terrestre entre el Valle de Aburrá y la salida al mar Caribe (Golfo de Urabá) por ello, los diferentes proyectos de infraestructura vial han impactado en su vida social generando cambios en el uso del suelo y con esto en el perfil de la población ha transitado de una economía agrícola campesina basada en la producción cafetera a una economía de servicios terciarios como restaurantes.

A nivel poblacional, el último Censo Nacional de Población y Vivienda del 2018 reporta una población total de 2.372.330 para Medellín, de este total, 39.843 habitantes en las zonas rurales $^{6}$ (DANE, 2018)

La población urbana se concentra en el costado norte de la ciudad - laderas oriental y occidental- y la población rural se considera como rural disperso, es decir su densidad poblacional es entre 1 y 10 habitantes por kilómetro cuadrado a diferencia de la densidad

\footnotetext{
${ }^{6}$ Dato ilustrativo debido a la ausencia de microdatos publicados por cada una de las cinco zonas rurales de la ciudad de Medellín.
} 
poblacional urbana que tiene rangos entre los 60 y 500 habitantes por kilómetro cuadrado (DANE, 2005)

Aunque la población rural es casi cincuenta veces menor que la población urbana, el área rural representa un territorio extenso, básico para el equilibrio ambiental del municipio y culturalmente significativo ya que los recursos naturales como bordes de la ciudad de Medellín son ecosistemas con una alta presión urbana, no solo a nivel de renta urbana, sino también como zonas de uso y consumo de la naturaleza.

\section{Metodología}

La metodología empleada se fundamentó en el enfoque de la investigación social cualitativa y la investigación participativa, adaptando técnicas como (i) la revisión documental, (ii) el sociograma7, (iii) y las entrevistas semiestructuradas.

El proceso consistió primero, en convocar a un taller a los integrantes de organizaciones sociales de base en cada uno de los cinco corregimientos, un taller por cada uno de los cinco corregimientos.

Segundo, se realizaron las entrevistas semi estructuradas a algunas de las personas participantes en los talleres acorde a la participación y representatividad de cada organización en el corregimiento.

Y, tercero, se realizó la sistematización, triangulación y análisis de los datos recolectados en cada uno de los talleres y en las entrevistas.

El proceso de investigación se realizó durante los años 2013, 2014 y 2015, cubriendo el período de presentación, debate y aprobación del Plan de Ordenamiento Territorial de Medellín bajo el decreto 268 del 2014.

Para realizar el sociograma y las entrevistas se usaron criterios éticos de investigación como la carta de consentimiento informado y la sustitución de nombres propios para custodiar la identidad personal de quienes participaron en forma voluntaria con cada uno de los testimonios recolectados y citados en este artículo por tanto, cada testimonio detalla atributos personales y organizativos de las personas, omitiendo la identidad personal y como garantía de la confidencialidad pactada en la carta de consentimiento informado, entre las personas entrevistadas y el grupo de investigación.

\footnotetext{
${ }^{7}$ Son representaciones gráficas de las relaciones que existen entre grupos, instituciones o personas (Villasante, 2006, p. 415).
} 
En detalle, las técnicas de investigación empleadas consistieron en, la revisión documental de artículos de revistas y de tesis de pregrado y postgrado de las universidades locales. Se empleo la búsqueda de estos documentos a partir de palabra claves como área rural-ordenamiento territorial - Medellín. De los resultados de estas búsquedas se realizó una selección de documentos emblemáticos para la investigación acorde a recurrencia de autores, instituciones que anidaron el aporte, enfoque teórico y metodológico. Esta técnica de investigación posibilitó en gran medida, el contacto y posteriores entrevistas con expertos en el tema y, por consiguiente, sus aportes en publicaciones y en testimonios fueron centrales en el proceso de investigación.

El sociograma se asumió como un mapa de redes formales e informales, es decir de actores sociales y los vínculos que los unen. Se diseñaron y adaptaron de acuerdo a (i) grado de participación de los tres tipos de actores sociales en el ordenamiento rural de la ciudad, se emplearon en el diseño, desarrollo y análisis los tres grupos de actores sociales que sugiere el Observatorio de Participación y Medio Ambiente CIMAS (actores organizados, actores institucionales y actores no organizados) (ii) las relaciones entre estos actores (iii) su poder simbólico u organizativo y (iv) la capacidad de influencia en el tema. (Villasante y Martin, 2007: 130).

Los sociogramas identifican conjuntos de acción, es decir conjuntos dinámicos de actores que se forman sobre tres variables: las redes de confianza en las comunidades, los condicionantes de clase social y las posiciones sobre el problema en disputa concreto. Estas variables se visualizan en el eje $x$ (horizontal), eje $y$ (vertical) y los puntos de intersección al interior del plano (Figura 2).

De esta técnica de sociograma surgieron los actores a entrevistar seleccionados por su vinculación en organizaciones de base o entidades con presencia y participación en cada una de las cinco zonas rurales de la ciudad.

Finalmente, las entrevistas semi estructuradas hacen parte de las técnicas de historia oral y en esta oportunidad, las entrevistas buscaban profundizar en los testimonios de las personas que participaron en los talleres de sociogramas en los cinco corregimientos. Se realizaron 22 entrevistas en total. Las personas entrevistadas cumplían perfiles como (i) hombres y mujeres expertos y (ii) hombres y mujeres adultos y jóvenes integrantes de organizaciones sociales de base.

Las personas seleccionadas para las entrevistas, en consonancia con el enfoque 
cualitativo de la investigación social, poseen atributos (cualidades) como rol de cohesión dentro de la organización y en el corregimiento, participación activa y regular y capacidad de gestión local. Las organizaciones que se identificaron corresponden a: medios de comunicación comunitarios, campesinos (con tierra o cultivos propios), campesinos (sin tierra, pero con procesos asociativos), organizaciones juveniles, colectivos de mujeres y/o de género, colectivos ambientales, empresas constructoras, instituciones ambientales, instituciones educativas y secretaría de medio ambiente y de planeación.

Las entrevistas por cada uno de los cinco corregimientos se realizaron cubriendo, en la medida de lo posible, cada uno de los perfiles y al llegar al punto de saturación (reiteración de datos por perfil y localidad) se dio cierre a la etapa de campo para proceder a sistematizar y diseñar la devolución de información por medio de eventos co organizados a nivel local (talleres de devoluciones creativas, según el Observatorio CIMAS)

Las entrevistas semi estructuras a expertos, en cambio y como se indicó antes, se realizaron antes y posterior a la etapa de campo bajo los criterios de selección por recurrencia en publicaciones en el ordenamiento territorial rural de la ciudad.

El proceso metodológico de la investigación posibilito el análisis de los discursos según tipo de actores sociales (organizados, no organizados e institucionales) primero, en forma gráfica en los sociogramas y luego, a partir de las posturas surgidas de las entrevistas.

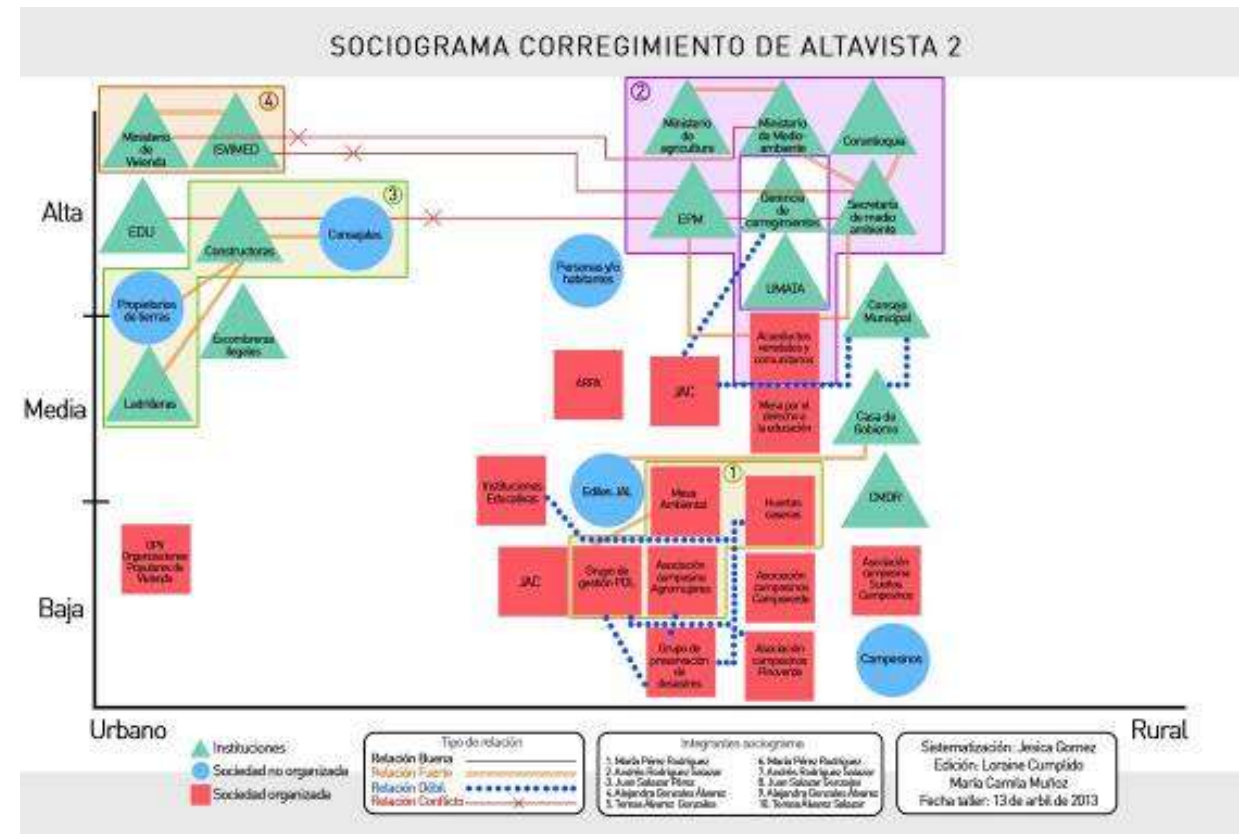

Figura 2. Sociograma del Corregimiento de San Cristóbal. Elaboración propia. Zona urbana del Corregimiento de Altavista, abril del 2013. Fuente: Grupo Medio Ambiente $\mathrm{y}$ Sociedad. 
Estos datos - posiciones y relaciones, discursos y testimonios - buscaban dar respuesta a la pregunta ¿Cuáles son los actores sociales y los discursos vinculados con el ordenamiento territorial rural de la ciudad de Medellín?

El eje $x$ (horizontal) señala el grado de interés y prácticas rural o urbana en la localidad. El eje y (vertical) indica la capacidad de influencia en la política de ordenamiento rural en la localidad (baja, media o alta). Cada forma geométrica (cuadro, circulo y triangulo) al interior identifica el tipo de actor social así, cuadros rojos son sociedad organizada, círculos azules son sociedad no organizada y triángulos verdes son instituciones.

Las líneas indican el tipo de relaciones que hay entre estos tres tipos de actores sociales, así: relación buena, línea negra continua; relación fuerte, línea amarilla; relación débil, línea punteada azul y, relación de conflicto, línea roja con asta.

En este sociograma se identifican así, 15 cuadros rojos (sociedad organizada), 14 triángulos verdes como instituciones y, 4 círculos de sociedad no organizada (propietarios de tierras-concejales-campesinas y población en general). Se reconoce la diversidad de actores organizados (colectivos mujeres campesinas, ambientales, huertas escolares, entre otros). Y, también presencia de varias instituciones.

A nivel de relaciones sobresalen las relaciones de conflicto (línea roja con asta) entre instituciones en el nivel de alto grado de influencia y, las relaciones fuertes y débiles (líneas amarillas y azules) entre actores organizados y con media y baja influencia en la política de ordenamiento rural de Medellín, según algunos fragmentos de la parte alta y baja del sociograma.

También, el sociograma indica varios conjuntos de acción, estos son las agrupaciones de las figuras geométricas como los círculos (actores sociales organizados) mantienen relaciones fuertes o débiles y, los triángulos (actores institucionales) tiene relaciones de conflicto. Los conjuntos de acción posibilitan la definición de estrategias colectivas para consensuar acciones concretas entre actores con posiciones antagónicas y lograr consensos mínimos. El sociograma, como técnica participativa, es importante para reconocer las tensiones (internas y externas) de las comunidades, pero también en los procesos de investigación más clásicos posibilitan una fotografía instantánea, sintética y temporal para comprender las situaciones reales y optimizar el tiempo y recursos de la investigación; es una técnica compatible con otras, como la entrevista semi estructurada. 


\section{Resultados}

Como se indicó en la metodología, la investigación busca identificar los actores sociales y los contenidos de sus discursos, en esta medida la recolección y análisis de los datos se organizaron y clasificaron en estas dos rutas, primero como actores y segundo, como discursos. A continuación, se presentan a nivel general, los principales y más recurrentes resultados, evitando una presentación detallada por cada uno de los cinco corregimientos debido a los límites editoriales del presente texto.

Los actores sociales ${ }^{8}$ vinculados con la política de ordenamiento territorial rural de Medellín se han clasificado acorde al objetivo y metodología del trabajo en tres grandes grupos: El primero corresponde a actores de las organizaciones de base, el segundo a las instituciones públicas y privadas y el tercero a la comunidad no organizada.

Los sociogramas han permitido ubicar en posiciones a los actores sociales y son estas posiciones -como lugares de enunciación- las que hacen posible encontrar una diversa gama de identidades locales en los corregimientos. Sobresalen por su regularidad en los sociogramas, los actores sociales de base. En este grupo se encuentran (i) campesinos (ii), obreros urbanos con residencia rural (iii) y habitantes neo rurales.

El campesino es el grupo de actores sociales donde se expresan en forma notoria una permanente identidad y vinculación con la vida del campo, referida en forma central a las actividades agrarias. Se caracteriza por un sentido del lugar marcado por la ancestralidad, la transferencia heredada de artes y oficios y por una vida cotidiana atada a los ciclos y ritmos naturales. Por ejemplo, lo que se expresa en el siguiente testimonio: "Acá hay una convivencia porque se conoce toda la familia, hay una tradición, una buena convivencia por ese mismo conocimiento de quién es el otro" (Mujer adulta, Corregimiento de San Sebastián de Palmitas, comunicación personal, 17 de septiembre del 2013).

El campesino se constituye en un actor social emblemático para los discursos que claman por conservar la tradición cultural. Pero los campesinos, en su discurso, están expresando con urgencia los efectos de los cambios en su vida cotidiana: aplauden las opciones y posibilidades del contacto con la ciudad a nivel del mercado y de los servicios como educación, salud y telecomunicaciones.

Para estos actores sociales su identidad local está determinada por la actividad agraria

\footnotetext{
${ }^{8}$ Una presentación y análisis detallado de actores sociales se encuentra en Egio y Torrejón, 2014 y

Pimienta y Torrejón, 2014.
} 
pero no se agota en ésta, se construye a la vez por una permanente relación social espacial y temporal con los otros, esto, por ejemplo, se expresa en el siguiente testimonio:

Yo me soñaría un corregimiento que integre distintas actividades económicas de beneficio para la comunidad y que se pudiera conservar la actividad agrícola dándole un manejo ambientalmente sostenible. Creo que sería una potencialidad para el municipio de Medellín si eso se pudiera hacer. Considero que esta vereda es clave para el corregimiento, porque tiene el "Alto del Padre Amaya" que es un ecosistema estratégico, es un páramo (Mujer adulta, Corregimiento de San Cristóbal, comunicación personal, 24 de septiembre del 2013).

Me imagino un turismo en las casas campesinas, pero un turismo diferente, no de venir y conocer solamente, sino como un turismo desde lo académico, que puedan venir aquí muchas personas con el fin de estudiar, conocer. Aquí hay zona rural y urbana (Mujer adulta, Corregimiento de San Cristóbal, comunicación personal, 24 de septiembre del 2013).

Quizás, y desde una visión fatalista, la identidad campesina se está perdiendo, pero desde una mirada distinta, quizás se están gestando unas nuevas identidades campesinas a la luz de las contingencias de la ciudad metropolitana, en términos de Luis Carlos Agudelo (2012), de un campesino metropolitano9 ${ }^{9}$, en este sentido se encuentra una visión (política) del campesino sin tener necesariamente tradición campesina:

Hay otro grupo grande de campesinos que, son personas que no viven en el campo permanentemente, pero están vinculados a procesos organizativos, simbólicos o culturales con las comunidades rurales. Mantienen un proceso vital por la defensa del campesinado, independiente de donde tenga su casa, son gente que viven y defienden la ruralidad (Hombre adulto, experto, en comunicación personal, 3 de septiembre del 2013)

El segundo grupo de actores es el obrero urbano con residencia rural. Este alude al conjunto de actores sociales que por causa de la oferta de empleo o de educación se trasladan en forma rutinaria a otros espacios diferentes y alejados de su residencia en la zona rural. Se caracterizan por su transectorialidad entre las zonas rurales y urbanas, por una cotidianidad fragmentada socio-espacial y temporalmente debido a las distancias y los modos de transporte que restan tiempo para la vida cotidiana y por su vinculación con los ritmos y ciclos laborales industriales. El obrero urbano vive en la ciudad, pero duerme en su casa

\footnotetext{
${ }^{9}$ Una presentación detallada del proceso metropolitano y los cambios de los perfiles poblacionales para algunas ciudades en América Latina son presentados en los aportes de Cruz (2003); Ramírez Velásquez (2003); Farah y Pérez (2004); Pérez (2004); Barros (2006); Llambí y Pérez (2007); Pradilla Cobos (2009) y Agudelo (2012)
} 
ubicada en la zona rural o asume algunas temporadas para residir en la ciudad, buscando ahorrar tiempo y dinero en el transporte.

El obrero urbano mantiene un contacto e identidad local, aunque sea en forma diferida. Cuenta con elementos identitarios de gran versatilidad, tanto para incorporarse y sobrevivir en el mercado laboral industrial (principalmente en construcción y servicios) como con las culturas campesinas -agrarias-, que ahora vivencia desde la distancia, desde una posición lugar de enunciación- ajena o, desde una paulatina pérdida. Esto se indica a partir de las condiciones de comunicación vial con los centros urbanos más cercanos, como se indica en el siguiente testimonio:

Aunque todo implique unos costos muy altos, porque acá desplazarse a la ciudad no es económico, tenerla a 40 minutos, una hora, es una ventaja. Tener una ciudad como Medellín, a 40 minutos de esto tan rural, eso es una gran ventaja, sin duda alguna; porque podemos acceder a la educación, a servicios, la gente puede ir a trabajar, volver al corregimiento (Mujer adulta, Corregimiento de San Sebastián de Palmita, comunicación personal, 24 de septiembre 2013).

La mayoría de personas se trasladan a San Cristóbal o al centro (de Medellín) a trabajar en distintos oficios, pues eso tiene que ver con la formación académica de las personas (Mujer adulta, Corregimiento de San Cristóbal, comunicación personal, 24 de septiembre del 2013).

Finalmente, entre los actores sociales organizados se encuentran los neo rurales, entendidos para este artículo como personas que habitan las zonas rurales, en principio, como estilo de vida. Un estilo de vida que se origina por la desilusión de la ciudad central en referencia a la pérdida de los atributos de la ciudad como obra máxima de la humanidad, en palabras de Lefebvre (1978)

Los neo rurales habitan las zonas rurales, espacializan su vida cotidiana a partir de sus conglomerados culturales, es decir desde sus experiencias anteriores, incluido el consumo de tecnología y de telecomunicaciones. El neo rural adapta el espacio rural para desarrollar su vida social vinculada con los servicios de telecomunicaciones, seguridad y estética. El siguiente testimonio evidencia estas características:

Para ellos el campo es una locación, y buscan ahí amenidades, pero no es un territorio, no es un corregimiento, pues, en el sentido completo del término. Es una locación con amenidades, eso también cuentan y, mucho, muchísimo en el proceso (Hombre adulto, experto, en comunicación personal, 3 de septiembre del 2013). 
Son actores sociales que depositan en las zonas rurales las promesas de una vida social más sosegada y cualitativamente mejor dotada de servicios ambientales: zonas verdes, agua, aire, flora y fauna. Su presencia y las relaciones socio espacio temporales que establecen en las zonas rurales pueden llevar a procesos de segregación espacial o bien a un interesante y prometedor intercambio cultural.

Ambas tendencias, la segregación espacial y/o el intercambio cultural dependerán de las formas de participación y de convivencia frente a los conflictos por el uso (privado y/o público) de lo rural: unos se podrán resolver y otros en cambio, aumentarán las desigualdades sociales. La integración cultural demanda en sí misma que las identidades locales absorban, mimeticen y transformen el encuentro de los diferentes estilos de vida en confluencia con los neo rurales y los nativos, generando así permanentes transacciones culturales, unas con saldo negativo (pérdidas del sentido del lugar) y, otras, con nuevos sentidos del lugar (nuevas identidades locales)

El segundo tipo de actores sociales, son las instituciones públicas y privadas. Sus discursos se pueden caracterizar por una permanente lectura del territorio rural como (i) un instrumento para la economía de escala de materias primas, (ii) espacio para el control y la regulación territorial (iii) un espacio para el modelo de desarrollo urbano y (iv) espacios sociales para la vida y la cultura campesina y ambiental ${ }^{10}$. Las instituciones como actores sociales son variadas por los niveles administrativos del Estado. Se encuentran entidades de autoridad local hasta nacional e instituciones privadas nacionales y, también, internacionales. Esta situación coincide con la etapa que actualmente se desarrolla como planeación multivariada, es decir, se presentan múltiples instrumentos de planeación local y, con éstos, múltiples actores sociales estatales en ocasiones con poca o ninguna coordinación. En el siguiente testimonio, por ejemplo, se ilustra la visión de la planeación que tiene uno de los habitantes de las zonas rurales:

La ciudad no consensa con el campo, la planeación, el ordenamiento y la recuperación del territorio. La ciudad urbana impone sobre el campo lo que hay que hacer, porque es quien tiene el recurso y el poder para hacerlo y el campo es indefenso (Hombre adulto, Corregimiento de San Antonio de Prado, comunicación personal, 24 de septiembre del 2014).

\footnotetext{
10 Se resalta el papel de La Corporación Ecológica y Cultural Penca de Sábila, organización de la sociedad civil que cuenta con una larga y determinante experiencia en el acompañamiento a la población rural campesina, además de fomentar los circuitos cortos como estrategia para la comercialización de la producción agrícola con prácticas limpias y comunitarias.
} 
"Entonces hay que ver que la planeación para la ciudad de Medellín no debe hacerse sólo desde la dirección central desde Alpujarra, debe hacerse decentralizadamente (Hombre adulto, docente, Corregimiento de San Antonio de Prado, comunicación personal, 24 de septiembre del 2014).

Finalmente, se encuentran los actores sociales no organizados, se considera en éstos a todo el conjunto de sujetos sociales que habitan los territorios rurales y que desarrollan su vida cotidiana en estos, aunque no participen de las organizaciones de base o participen de otras organizaciones en diferentes formas.

Así, desde esta tipificación de actores sociales se encuentra una marcada presencia participativa de ellos en las zonas rurales de Medellín, desdibujando la idea de una zona rural sin participación en el mapa de la ciudad donde cada corregimiento tributa con una participación activa de actores sociales a la construcción de identidades locales

\section{Reflexiones finales}

Los actores sociales que se han enunciado en este artículo y cada discurso alrededor de la política de ordenamiento territorial rural en Medellín permiten dar las respuestas a la pregunta de investigación ¿Cuáles son los actores sociales y los discursos vinculados con el ordenamiento territorial rural de la ciudad de Medellín?

Primero, hay un conjunto de actores sociales organizados (campesinos, obreros urbanos y neorurales) dispersos en la extensa área rural de Medellín -270km2-. Sus discursos son variados, creativos y diversos como fuerza social que, parece, pudiese resistir frente a las turbulencias de una ciudad metropolitana en la globalización. Esta fuerza social se manifiesta en la localidad como una construcción social y se alimenta de materiales intangibles (sentidos del lugar) base de una región como conexión de lugares (Albet, 2001) que emerge alrededor de las identidades locales de las zonas de borde, periféricas y marginales de la ciudad. Estas fuerzas sociales son las que han realizado el seguimiento al Plan de Ordenamiento Territorial y las que, en la actualidad, realizan mayor presión social para la implementación del Distrito Rural Campesino, entre otras demandas ante los gobiernos locales de turno.

Segundo, los discursos de los actores sociales (organizados, no organizados e instituciones) están usando elementos emblemáticos que transitan desde una visión de lo rural tradicional hasta lo rural moderno. En este tránsito hay discursos espaciales que están modelando el desarrollo territorial de las cinco zonas rurales de la ciudad: unos desde una 
profunda y arraigada convicción del papel del campesino en lo local (como sujeto de derechos) y, otros, desde una llamativa reconstrucción del papel del campesinado en la ciudad metropolitana (también, como sujeto de derechos)

Entre ambas tendencias discursivas hay un elemento común: la crítica al modelo de desarrollo urbano expansivo de Medellín y un urgente llamado por una justicia espacial donde se reconozcan las identidades locales desde las particularidades de cada una de las cinco zonas rurales de la ciudad como bienes públicos y patrimonio cultural.

Los discursos presentan una gama de posiciones entre lo rural y lo urbano, mediando entre éstos una tendencia a (i) mantener las zonas rurales por su carácter particular, singular y periférico, como lugar (ii) garantizar a los habitantes rurales el acceso universal a los servicios sociales como salud, educación, empleo, transporte, telecomunicaciones, recreación y acceso al derecho de propiedad de la tierra11, como sentido global del lugar y (iii) como justicia espacial y bien común, al valorar y compensar los costos y beneficios de las funciones ambientales y socioculturales que cumplen en la actualidad las zonas rurales para la ciudad, es decir, como co gestión de los bienes comunes.

En este artículo se presentaron los tipos de actores sociales y los discursos más emblemáticos en el ordenamiento territorial de las zonas rurales de Medellín, se asumió la relación entre la justicia espacial, el sentido del lugar y los bienes comunes como ruta analítica para reconocer las características e identidades locales emergentes desde las organizaciones sociales de las ruralidades de la ciudad de Medellín y, su papel en los procesos participativos a la hora de realizar y consensuar un ordenamiento territorial rural para y con la gente.

Más allá de los 270 kilómetros cuadrados de extensión de la zona rural y los procesos de ordenamiento territorial que se reúnen en estas áreas, los cinco corregimientos son también y, principalmente, espacios representativos de apropiación social colectiva para la defensa de los recursos naturales y culturales de Medellín, son bienes comunes que requieren auto gestión local frente a la presión de uso del espacio público verde de los casi casi tres millones de habitantes del perímetro urbano.

Esta presión de espacio público verde a colapsado la capacidad de carga de zonas como Santa Elena, zona rural que ha contado con la instalación de mega estructuras como el Metro

\footnotetext{
11 Se considera la realización e implementación del Plan de Ordenamiento Social de la Propiedad Rural, como una de las estrategias para proteger los derechos de acceso y tenencia de la tierra
} 
Cable Arví12 y, con esto con el eventual aumento de la renta del suelo a partir de la inversión de capital público y privado.

Esta presión urbana tiene relación directa con el aumento, en las últimas dos décadas, de la presión del suelo en las zonas rurales para vivienda, turismo y servicios ambientales. A esto hay que sumar, por su condición geográfica de zonas de borde, la necesidad de disponer de corredores de transporte para facilitar la comunicación de Medellín con otras regiones tanto del departamento, como de Colombia, de Latinoamérica y del resto del mundo, como se evidencia en los casos de los corregimientos de San Cristóbal y de San Sebastián de Palmitas, al noroeste de la ciudad.

Las organizaciones de base tanto de los cinco corregimientos de la ciudad como de los colectivos sociales del perímetro urbano han posibilitado, a partir de la presión social y de la auto gestión ambiental, la revalorización de lo rural en una ciudad como Medellín que presenta una desigual distribución de la renta e indicadores como la tasa de homicidios, aún bastante significativos y en contraste con la promoción y publicidad de los últimos gobiernos locales bajo la campaña de la transformación urbana de Medellín (Daniel Quintero13, 20202022, Federico Gutiérrez, 2016-2019, Aníbal Gaviria 2012-2015, Alfonso Salazar 2008-2011 y Sergio Fajardo 2004-2007).

En el caso de las zonas rurales de Medellín, aparecen actores sociales y discursos que revierten la geometría del poder en términos de Massey al determinar sus identidades locales a partir de una conciencia espacial -geográfica - periférica, de borde o marginal. Los derechos de acceso a servicios básicos (incluyendo, por ejemplo, la información y acceso a internet universal), la equidad en la distribución o retribución de la renta por servicios ambientales como espacio público verde, paisaje y otros servicios ecosistémicos vinculados a la red hídrica y el sistema agroalimentario de la ciudad región. Es decir, al derecho a la ciudad según Lefebvre, el derecho a la co-gestión de los bienes comunes acorde a Capel (2003) y una justicia espacial desde sus prácticas locales según Soja (2010)

En síntesis, la conciencia espacial de los pobladores de las zonas rurales presenta características comunes: (i) se rescatan las culturas campesinas (andinas), (ii) se re significan los servicios ambientales como bienes comunes de la población de la ciudad de Medellín,

\footnotetext{
12 Una de los modos de transporte asociados al sistema integrado de transporte público de la ciudad que se ha estructurado a partir del funcionamiento del Metro Elevado desde la década de 1990.

13 Electo bajo los resultados del 27 de octubre del 2019
} 
localizados principalmente en las cinco zonas rurales y,(iii) se promueve instrumentos de planeación como el Distrito Rural Campesino, como una de las formas de auto gestión y de defensa de los derechos territoriales colectivos centrado en el hábitat y culturas campesinos en una escalar local y global, con un sentido global del lugar.

\section{Referencias}

Acosta, A. (2010). El Buen Vivir en el camino del post-desarrollo. Una lectura desde la Constitución de Montecristi. Policy Paper, 1-36.

Acosta, A. (2014). Post-crecimiento y post-extractivismo: dos caras de la misma transformación cultural. En G. Endara, Post-crecimiento y buen vivir (pp. 95-124). Ecuador, Quito: Friedrich-Ebert-Stiftung (FES-ILDIS).

Arango, G. (2008). Salvemos el espacio rur urbano colombiano. Cuadernos de vivienda y urbanismo, 1(2).

Agudelo, L. (2012) Ruralidad Metropolitana. Entre la tradición rural y el “brillo" urbano. Una interpretación. Buletin d L'Institut Francs d'Études Andinos, 4 (3), 555-571.

Albet i Mas, A. (2001). ¿Regiones singulares y regiones sin lugares? Reconsiderando el estudio de lo regional y lo local en el contexto de la geografía postmoderna. Boletín de la Asociación de Geógrafos Españoles, 32, 35-52.

Albet, A. y Bénach, N. (2012). Doreen Massey. Un sentido global del lugar. Espacios Críticos, Barcelona, España: Icaria.

Barros, C. (2006). La ciudad en el campo: Nuevas ruralidades y lugares rur urbanos, En: Nogué, J. y Romero, J. (Ed.), Las otras geografías (pp.325-228). Valencia, España: Tirant Lo Blanch.

Boof, L. (1996). Ecología, grito de la tierra, grito de los pobres. Mexico D.F: Ediciones Dabar. 
Capel, H. (2003). El drama de los bienes comunes. La necesidad de un programa de investigación. Revista Bibliográfica de Geografía y Ciencias Sociales, 8 (458).

Cruz, M. (2003). Presentación. Sociológica. Revista del departamento de sociología (51), 5-10. Recuperado de: http://www.revistasociologica.com.mx/pdf/5101.pdf. (Consulta el 10/9/2009)

Cadavid, G. (2009). Ruralidad en contextos metropolitanos, un desafío en procesos de planeación, ordenamiento territorial y gestión. Revista Soluciones de Postgrado EIA, (4), 243266. Medellín: Escuela de Ingeniería de Antioquia.

Castaño, Y. y Agudelo, L. (2014) Rurales Metropolitanos: La Emergencia de un Nuevo Grupo Social. Tesis de Maestría en Planeación Urbano Regional. Universidad Nacional de Colombia. Escuela de Planeación Urbano Regional.

Departamento Nacional de Estadística (2005). Censo Nacional de Población y Vivienda. Recuperado de: http://www.dane.gov.co

Departamento Nacional de Estadística (2018). Censo Nacional de Población y Vivienda. Recuperado de: http://www.dane.gov.co

Egio, C. J. y Torrejón, E. Y. (2014). Actores sociales y ordenamiento territorial rural los corregimientos de Medellín, Colombia. Bitácora Urbano Territorial, 24(2), 151-160. Recuperado de: http://www.scielo.org.co/scielo.php?script=sci_arttext\&pid=S012479132014000200151\&lng=en\&tlng=

Escobar, A. (1999a). Antropología y desarrollo. Maguare, 14, 42-73.

Escobar, A. (1999b). Cultura, ambiente y política en la antropología contemporánea. Instituto colombiano de antropología, Ministerio de Cultura.

Farah, M. y Pérez, E. (2004). Mujeres rurales y nueva ruralidad en Colombia. Cuadernos de 
desarrollo rural (51). Bogotá, Colombia: Pontificia Universidad Javeriana.

García, E. y Gálvez, A. (2011). La gestión pública ambiental rural. Revista gestión y ambiente, 14 (2). Medellín, Colombia.

Gudynas, E. (2011). Desarrollo y sustentabilidad ambiental: diversidad de posturas, tensiones persistentes. En: Matarán, A. y López, F. (Ed.), La Tierra no es muda: diálogos entre el desarrollo sostenible y el postdesarrollo (pp. 69-96). Granada, España: Universidad de Granada.

Lefebvre, H. (1978). El derecho a la ciudad. España: Ediciones Península.

Lefebvre, H. (1983). La presencia y la ausencia. Contribución a la teoría de las representaciones. México: Fondo de Cultura Económica.

Llambí, L. y Pérez, E. (2007). Nuevas ruralidades y viejos campesinismos. Agenda para una nueva sociología rural en América Latina. Cuadernos de desarrollo rural (59), 37-61. Bogotá: Pontificia Universidad Javeriana.

Municipio de Medellín (1999). Acuerdo Municipal 62 de 1999. Plan de Ordenamiento Territorial de Medellín. Medellín: Alcaldía de Medellín.

Municipio de Medellín (2005). Departamento Administrativo de planeación. Estrategias Corregimentales para Medellín: ECO. Municipio de Medellín. Recuperado de: http://www.bdigital.unal.edu.co/9613/1/Eco.pdf

Municipio de Medellín (2006). Acuerdo Municipal 46 de 2006. Plan de Ordenamiento Territorial del Medellín. Medellín: Alcaldía de Medellín.

Municipio de Medellín (2014). Acuerdo Municipal 48 del 2014. Revisión y ajuste de largo plazo del Plan de Ordenamiento Territorial del Municipio de Medellín. Medellín: Alcaldía de Medellín. 
Pradilla Cobos, E. (2009). Campo y ciudad en el capitalismo actual. En: Los territorios del neoliberalismo en América Latina. Compilación de Ensayos (pp. 219-250). Xochimilco, México: UAM.

Pérez Correa, E. (2004). El mundo rural latinoamericano y la nueva ruralidad. Revista Nómadas, Universidad Central, Bogotá, 20 (1), 180-193.

Pimienta, J. y Torrejón, E. (2014). Análisis de Discurso de la Política Pública de Ruralidad en el Municipio de Medellín (2006-2012). Tesis de Pregrado de Sociología. Universidad de Antioquia. Medellín.

Ramírez Velásquez, B. (2003). La vieja agricultura y la nueva ruralidad: enfoques y categorías desde el urbanismo y la sociología rural. Sociológica, 8 (51), 49-71.

Soja, E. (2010). Seeking Spatial Justice. Minneapolis: Minnesota University.

Villasante, T. (2006). Desbordes creativos. Estilos y estrategias para la transformación social. Madrid, España: Catarata.

Villasante, T. y Martín, P. (2007). Redes y conjuntos de acción: para aplicaciones estratégicas en los tiempos de la complejidad social. Política y Sociedad, 1 (44), 125-140.

\section{Financiación}

Con recursos del Comité de Investigación Universitario Estrategia de Sostenibilidad de Grupos (versión 2019). Contó con el apoyo del Observatorio Internacional de Ciudadanía y Medio Ambiente Sostenible -CIMAS 